tion holds, as may be verified by direct substitution of $\xi=1$ in the general equations.

\section{ADDENDUM (Received 28 March 1951)}

The authors have recently received from R. E. Stoner of the Pennsylvania State College a shadowgram of the refraction of a shock wave at an interface separating air and carbon dioxide in a shock tube. With the kind permission of Professor Stoner, this shadowgram is reproduced here (Fig. 9). The measured angles produced by the interaction agree qualitatively with the theoretically predicted values. ${ }^{9}$

${ }^{9}$ R. G. Stoner and M. H. Glauberman, Phys. Rev. 76, 882 (1949).

\title{
Approximation Methods in the Theory of Scattering
}

\author{
MARVIN L. GolDBERGER \\ Institute for Nuclear Studies, University of Chicago, Chicago, Illinois
}

(Received July 9, 1951)

\begin{abstract}
The quantum theory of scattering is discussed from the point of view of a time independent formulation. A detailed discussion of the replacement of the ordinary integral equation of scattering by two others is given together with a discussion of the desirability of this procedure both from a physical and mathematical point of view. Variational formulations of these equations are given and their accuracy is indicated by comparison with examples for which exact solutions are known.

An exactly soluble problem given by Blatt is treated from the standpoint of the present paper. A formal solution of the general scattering problem is developed and illustrated with Blatt's example.
\end{abstract}

\section{INTRDOUCTION}

$\mathrm{O}^{\mathrm{s}}$ $\mathrm{NE}$ of the most important problems in physics is the determination of the various matrix elements of the so-called Heisenberg $S$-matrix. ${ }^{1}$ A variety of approximation methods have been used in practical calculations of these matrix elements. The types of problems studied range from nonrelativistic nucleonnucleon scattering with static potentials to very complex field theoretical problems which are beset by divergence difficulties. The general approach to all of these problems has been a perturbation theoretic one based on a succession of Born approximations. (The nonrelativistic scattering of nucleons by a static potential is the lone exception for which an exact solution is feasible.) Actually the Heitler theory of radiation damping, ${ }^{2}$ which will be discussed in detail later, is not a perturbation method in that it does not yield a power series in an interaction parameter. Recently, variational methods have been introduced for the treatment of scattering problems ${ }^{3}$ but as yet only a few simple problems have been studied with this method. Their chief importance is that they provide at least in principle a method for obtaining solutions which are not power series expansions of the variety obtained by direct iteration (successive Born approximations).

\footnotetext{
1 J. A. Wheeler, Phys. Rev. 52, 1107 (1937); W. Heisenberg, Z. Physik 120, 513, 673 (1943).

${ }^{2}$ W. Heitler, Proc. Cambridge Phil. Soc. 37, 291 (1941); A. H Wilson, Proc. Cambridge Phil. Soc. 37, 301 (1941); S. T. Ma and C. F. Hsuek, Proc. Cambridge Phil. Soc. 40, 167 (1944).

${ }^{3}$ J. Schwinger, Lectures on Nuclear Physics (Harvard University, Cambridge, 1947); W. Kohn, Phys. Rev. 74, 1763 (1948); L. Hulthen, see Mott and Massey, The Theory of Atomic Colli sions (Oxford University Press, London, 1949), second edition p. 128.
}

The purpose of the present work is to discuss in greater detail the results of a previous paper, ${ }^{4}$ to give examples of the use of the variational methods developed there, and finally to discuss the importance of treating separately, in so far as it is possible, the inductive and resistive parts of the field reactions. This work was begun in connection with a study of the paper by Pais and Jost ${ }^{5}$ on the failure of the second Born approximation as applied to non-relativistic nucleonnucleon scattering. The methods discussed below have been applied to this problem and the results already obtained are encouraging. This work is being continued by Messrs. A. Kaufman and F. Solmitz and will be reported soon. The problems to be treated in the present paper are all concerned with applications to field theories.

\section{DERIVATION OF THE FORMULAS}

A fairly complete derivation of the formulas to be used in this paper was given in I but for ease of reference the principal results will be restated here. In addition it will be shown that the results given there are true under much more general conditions than were previously stated. We shall again follow the development of Lippmann and Schwinger ${ }^{6}$ with the previously noted ${ }^{4}$ changes in notation.

We wish to solve the Schroedinger equation,

$$
\left(E-H_{0}\right) \Psi=H_{1} \Psi
$$

subject to certain boundary conditions. These are taken

\footnotetext{
${ }^{4}$ M. L. Goldberger, Phys. Rev. 82, 757 (1951), referred to hereafter as $I$.

${ }^{5}$ R. Jost and A. Pais, Phys. Rev. 83, 840 (1951).

B B. Lippmann and J. Schwinger, Phys. Rev. 79, 469 (1950).
} 
to be an incident "plane wave" (i.e., solution of the problem with $H_{1}=0$ ) plus either incoming or outgoing scattered waves. We replace (2.1) by an integral equation which incorporates these boundary conditions:

$$
\Psi_{a}^{( \pm)}=\Phi_{a}+\frac{1}{E_{a} \pm i \epsilon-H_{0}} H_{1} \Psi_{a}^{( \pm)}
$$

where $\Phi_{a}$ is an eigenfunction of $H_{0}$ corresponding to the energy $E_{a}$ and represents the initial state of the system. The transition probability per unit time from the state characterized by $\Phi_{a}$ to an initially unoccupied state $\Phi_{b}$ is given by

$$
w_{b a}=(2 \pi / h)\left|\mathbf{R}_{b a}\right|^{2} \delta\left(E_{b}-E_{a}\right),
$$

where the matrix $\mathbf{R}$ is defined only for states of equal energy. Its definition is

$$
\mathbf{R}_{b a}=-\left(\Phi_{b}, H_{1} \Psi_{a}^{(+)}\right)=-\left(\Psi_{b}^{(-)}, H_{1} \Phi_{a}\right),
$$

with $E_{a}=E_{b}$. We shall later have use for the total rate of transition from the state $\Phi_{a}$ which may be written as

$$
\sum_{b w_{b a}}=(2 / \hbar) \operatorname{Im} \mathbf{R}_{a a} .
$$

This is based on the unitarity of the $S$-matrix which is related to $\mathbf{R}$ via

$$
S_{b a}=\delta_{b a}+2 \pi i \mathbf{R}_{b a} \delta\left(E_{b}-E_{a}\right) .
$$

A variational formulation of (2.2) together with applications is given in reference 6 . We now pass on to discuss in detail the considerations given in $I$.

Instead of solving (2.1) in terms of scattered waves as in (2.2) it is convenient to introduce a "standing wave" solution which contains equal amounts of incoming and outgoing scattered waves. This is achieved by noting that

$$
\frac{1}{2}\left[\frac{1}{E_{a}+i \epsilon-H_{0}}+\frac{1}{E_{a}-i \epsilon-H_{0}}\right]=\frac{P}{E_{a}-H_{0}},
$$

where $P$ stands for the Cauchy principal value. In terms of this Green's function, an integral equation incorporating (2.1) and the desired boundary conditions may be written as

$$
\Psi_{a}^{(1)}=\Phi_{a}+\frac{P}{E_{a}-H_{0}} H_{1} \Psi_{a}^{(1)} .
$$

We now adopt the notational convention that subscripts $a, b, c$ will be used to designate states of the same energy $E_{a}=E_{b}=E_{c}=E$, and subscripts $\alpha, \beta$ denote states for which $E_{\alpha}, E_{\beta} \neq E$. Forming the "radiation field" $\Psi_{a}{ }^{(+)}-\Psi_{a}{ }^{(1)}$ we have

$$
\begin{aligned}
\Psi_{a}^{(+)}-\Psi_{a}^{(1)}=\frac{P}{E_{a}-H_{0}} & H_{1}\left(\Psi_{a}^{(+)}-\Psi_{a}^{(1)}\right) \\
& +i \pi \sum_{c} \Phi_{c} \delta\left(E_{a}-E_{c}\right) \mathbf{R}_{c a},
\end{aligned}
$$

which, using (2.7) to eliminate $\Phi_{c}$ may be written as

$$
\begin{aligned}
\Psi_{a}^{(+)}-\Psi_{a}^{(1)}-i \pi \sum_{c} \Psi_{c}^{(1)} \delta\left(E_{a}-E_{c}\right) \mathbf{R}_{c a} \\
=P \frac{1}{E_{a}-H_{0}} H_{1}\left\{\Psi_{a}^{(+)}-\Psi_{a}^{(1)}\right. \\
\left.\quad \quad-i \pi \sum_{c} \Psi_{c}^{(1)} \delta\left(E_{a}-E_{c}\right) \mathbf{R}_{c a}\right\}
\end{aligned}
$$

From (2.9) it follows that

$$
\Psi_{a}{ }^{(+)}=\Psi_{a}^{(1)}+i \pi \sum_{c} \Psi_{c}^{(1)} \delta\left(E-E_{c}\right) \mathbf{R}_{c a}
$$

provided we assume that the operator

$$
\frac{P}{E_{a}-H_{0}} H_{1}
$$

has no eigenfunction corresponding to the eigenvalue unity. Equation (2.10) is thus a sufficient condition for the pair of Eqs. (2.7) and (2.10) to be equivalent to (2.2). Our final step is the introduction of the reaction matrix, $G$, according to the definition

$$
G_{\beta a}=-\left(\Phi_{\beta}, H_{1} \Psi_{a}^{(1)}\right) .
$$

We shall prove that $G_{\beta a}$ is not an hermitian matrix provided, as implied by our notation, $E_{\beta} \neq E_{a}=E$. This may be done by using (2.7) to eliminate $\Phi_{\beta}$

$$
\begin{aligned}
-G_{\beta a}= & \left(\Psi_{\beta}^{(1)}, H_{1} \Psi_{a}^{(1)}\right)-\left(\frac{P}{E_{\beta}-H_{0}} H_{1} \Psi_{\beta}^{(1)}, H_{1} \Psi_{a}^{(1)}\right) \\
= & \left(H_{1} \Psi_{\beta}^{(1)},\left\{\Psi_{a}^{(1)}-\frac{P}{E_{a}-H_{0}} H_{1} \Psi_{a}^{(1)}\right\}\right) \\
& +\left(H_{1} \Psi_{\beta}^{(1)},\left\{\frac{P}{E_{a}-H_{0}}-\frac{P}{E_{\beta}-H_{0}}\right\} H_{1} \Psi_{a}^{(1)}\right) \\
= & -G_{\beta a^{\dagger}}^{\dagger}+\left(E_{\beta}-E_{a}\right) \\
& \times\left(\Psi_{\beta}^{(1)}, H_{1} \frac{P}{\left(E_{a}-H_{0}\right)\left(E_{\beta}-H_{0}\right)} H_{1} \Psi_{a}^{(1)}\right) .
\end{aligned}
$$

Dividing by $E_{\beta}-E_{a}$ and allowing $E_{\beta}$ to approach $E_{a}$, we derive

$$
\left.\frac{\partial G_{\beta a}}{\partial E_{\beta}}\right|_{E_{\beta}=E_{a}}=-\left.P \sum_{\alpha} \frac{G_{\beta \alpha}^{\dagger} G_{\alpha a}}{\left(E_{\alpha}-E_{\alpha}\right)^{2}}\right|_{E_{\beta}=E_{\alpha}} .
$$

This equation may be used for approximate evaluations of $G_{\beta a}$ in the neighborhood of the energy $E_{\beta}=E_{a}$ by using for $G$ on the right hand side the Born approximation value, $G_{\beta \alpha} \simeq B_{\beta \alpha} \equiv-\left(\Phi_{\beta}, H^{\left(\Phi_{\alpha}\right.}\right)$.

Our fundamental equations are now obtained by substituting (2.7) and (2.10) into (2.11) and (2.4) re- 
spectively. We find

$$
\begin{aligned}
& G_{\beta a}=B_{\beta a}-P \sum_{\alpha} \frac{B_{\beta \alpha} G_{\alpha a}}{E_{a}-E_{\alpha}},\left[B_{\beta \alpha}=-\left(\Phi_{\beta}, H_{1} \Phi_{\alpha}\right)\right] \\
& \mathbf{R}_{b a}=\mathbf{G}_{b a}+i \pi \sum_{c} \mathbf{G}_{b c} \delta\left(E-E_{c}\right) \mathbf{R}_{c a} .
\end{aligned}
$$

Before discussing these equations, we give the analogue of (2.10) that deals with $\Psi_{b}^{(-)}$, namely,

$$
\Psi_{b}^{(-)}=\Psi_{b}^{(1)}-i \pi \sum_{c} \Psi_{c}^{(1)} \delta\left(E-E_{c}\right) \mathbf{R}_{b c}{ }^{*} .
$$

When this expression is substituted into (2.4) we obtain an alternate version of $(2.15)$ :

$$
\mathbf{R}_{b a}=\mathbf{G}_{b a}+i \pi \sum_{c} \mathbf{R}_{b c} \delta\left(E-E_{c}\right) \mathbf{G}_{c a} .
$$

This shows that $\mathbf{R}$ and $\mathbf{G}$ commute. This is a well known result which is usually ${ }^{6}$ given in the form of remarking that if $\mathbf{G}_{b a}$ be written in the diagonal form

with

$$
\mathbf{G}_{b a}=\sum_{A} f_{b A} G_{A} f_{a A} *
$$

$$
\sum_{a} f_{a A^{*}}{ }^{*} \delta\left(E-E_{a}\right) f_{a A^{\prime}}=\delta_{A A^{\prime}},
$$

$\mathbf{R}_{b a}$ may be written as

with

$$
\mathbf{R}_{b a}=\sum_{A} f_{b A} R_{A} f_{a A} *
$$

$$
R_{A}=G_{A} /\left(1-i \pi G_{A}\right)
$$

In other words, the eigenvectors of $\mathbf{G}$ are simultaneously eigenvectors of $\mathbf{R}$ and consequently $\mathbf{R}$ and $\mathbf{G}$ commute.

\section{DISCUSSION OF THE FORMULATION}

The above derivation of (2.14) and (2.15) is essentially the same as that given in a lecture by Schwinger, ${ }^{3}$ and is also partly contained in reference 6 . It has been given in some detail because the emphasis is somewhat different and to enable us to bring out a few important points, notably the non-hermitian nature of $G$ and the concomitant relation (2.13). Furthermore it is important to understand clearly that the values of $G$ off the energy shell are needed for its computation on the energy shell; this is evident from (2.14). The derivation of (2.15) given by Pauli ${ }^{7}$ also makes this point clear; Pauli's development suffers from the fact that $G$ is defined only by the iterated solution of (2.14). The resulting series rarely converges whereas the solution may very well exist, or at worst may exist after the now conventional renormalization processes are carried out.

Equation (2.15) is generally known as the Heitler integral equation ${ }^{2}$ although the term is frequently applied to the equation which results from replacing $G$ by $B$ (or the first nonvanishing iteration approximation to $G$, in general). With this approximation, it has been studied in detail by Heitler and his collaborators and forms the basis of the Heitler theory of radiation

\footnotetext{
${ }^{7}$ W. Pauli, Meson Theory of Nuclear Forces (Interscience Publishers, New York, 1946), Chapter IV.
}

damping. This theory has both defects and virtues which will be discussed later.

One may well question the advisability of replacing the single integral Eq. (2.2) by the pair of Eqs. (2.14) and (2.15). There are several arguments in favor of the procedure. First, the form of the equations enables one to see quite clearly the difficulties associated with the problem of the determination of interaction from scattering experiments. For suppose $\mathbf{R}_{b a}$ were known from experiment for all states $a, b$ for which $E_{a}=E_{b}=E$; then, in principle, (2.15) could be solved for $\mathbf{G}_{b a}$. The matrix $\mathbf{G}$ then determined is not however the matrix $G$ which is the one needed to enable one to solve (2.14) for $B$ in terms of $G$. $\mathbf{G}$ as determined from scattering experiments has matrix elements only between states of equal energies which leaves undetermined the "off energy shell diagonal" matrix elements needed in (2.14). Without some additional rules for the computation of the off diagonal elements of $G$ it would seem in general impossible to determine $B$ uniquely from scattering experiments. Second, a clean separation is made into quantities on and off the energy shell. This is more than an esthetic advantage; the types of process involved are of an essentially different nature. Basically, the Heitler equation gives an expression of the true radiation damping processes in the classical sense of reactions due to real processes (e.g., the reaction of an emitted photon on an accelerated electron). Equation (2.14) on the other hand, since it is not restricted to the energy shell, describes the virtual processes and is closely connected with self energy problems. Generally speaking, the divergence difficulties are isolated in the equation for $G$; if $G$ is finite, so is the resulting $\mathbf{R}$. Furthermore, if $\mathbf{R}$ be computed from (2.15) with any $\mathbf{G}$, the unitarity of $S$ is maintained, which is certainly very desirable. This is an argument in favor of even the rather radical Heitler damping theory: One does not obtain obviously nonsensical cross sections which increase indefinitely with energy, etc. What appears to be even more important is that even with the crudest $G$, namely $B$, an $\mathbf{R}$ derived from solution of (2.15) does contain some correct higher order effects.

There are of course many, perhaps equally significant, higher order corrections which are omitted by the neglect of higher order terms in $G$ and we are not advocating such a procedure; on the other hand, the damping effects are real and would appear in an exact solution of the problem. It would seem reasonable to take them into account in so far as one is able by a solution of (2.15).

There are cases where the neglect of higher order terms in $G$ is simply incorrect. The most prominent case is in the problem of electron scattering taking into account radiative corrections, which was treated by Bethe and Oppenheimer. ${ }^{8}$ It has been shown by Sebe ${ }^{9}$ that the inclusion of virtual photons in $G$ removes the

\footnotetext{
${ }^{8}$ H. A. Bethe and J. R. Oppenheimer, Phys. Rev. 70, 451 (1946). ${ }^{9}$ T. Sebe, Prog. Theor. Phys. 3, 304 (1948).
} 
discrepancy found in reference 9 . Blatt ${ }^{10}$ has shown that for a few simple problems the neglect of the higher order terms would appear to be numerically unjustified in that the neglected terms are larger than those retained. The general situation in field theories is, of course, greatly complicated by the appearance of divergences and it is difficult to draw definite conclusions from formal manipulations. Setting the divergence troubles aside for a moment, however, it would seem that an approximation procedure based on approximate solution of (2.14) together with an exact solution of (2.15), if possible, might yield quite good answers. For example, in problems involving the emission of high energy quanta (photons, mesons, etc.) the real damping effects may be expected to be large; a direct iteration of (2.2), say, might easily diverge. On the other hand the iteration procedure as applied to $G$, because of the energy denominators might even be convergent. The separation of the problem, which we propose, would then be very advantageous. With regard to divergences, we are certainly no worse off than we usually are. Presumably the renormalization program would be applied to $G$ in much the same way as it has been used for the $S$-matrix. It has been pointed out to the author by S. T. Ma that a similar proposal has been put forth by Fukuda and Miyazima. ${ }^{11}$

The problem of obtaining exact solutions to (2.15) is not a trivial one although it is a considerably simpler integral equation than (2.2) or (2.14) in that all quantities are restricted to the energy shell. It will be shown below that an iteration procedure is very likely to be only slowly convergent, if at all, and consequently different methods must be devised. What might be termed a semi-variational procedure has been proposed by $\mathrm{Ma}$ and Hsueh $;^{12}$ this will be discussed below. Only a few applications were made by those authors and the agreement with exact calculations was not too satisfactory. For this reason, and because of the importance of obtaining accurate solutions, we have in vestigated this problem and have found a more satisfactory variational formulation. In addition, a variational formulation of (2.14) has been found. In the next two sections the variational principles, briefly reported in I, will be discussed and examples of their utility given.

\section{VARIATIONAL FORMULATION}

We shall take up first the variational formulation of (2.15). It is convenient to introduce a notation for matrix multiplication for operators defined only on the energy shell, namely,

$$
(\mathbf{M N})_{b a} \equiv \sum_{c} \mathbf{M}_{b c} \delta\left(E-E_{c}\right) \mathbf{N}_{c a}, \quad E_{a}=E_{b}=E .
$$

We then assert that the matrix (on the energy shell),

\footnotetext{
${ }^{10}$ J. M. Blatt, Phys. Rev. 72, 466 (1947)

$11 \mathrm{~N}$. Fukuda and T. Miyazima, Prog. Theor. Phys. 5, 849 (1950).

${ }^{12}$ S. T. Ma and C. F. Hsueh, Phys. Rev. 67, 303 (1945).
}

$\mathfrak{3}$, with

$$
\mathfrak{I}=\mathbf{R} \mathbf{G}+\mathbf{G R}+i \pi \mathbf{R} \mathbf{G} \mathbf{R}-\mathbf{R} \mathbf{R}
$$

is stationary under arbitrary variations of $\mathbf{R}$. Performing such a variation, we obtain

$$
\delta \mathfrak{I}=\delta \mathbf{R}\{\mathbf{G}+i \pi \mathbf{R} \mathbf{G}-\mathbf{R}\}+\{\mathbf{G}+i \pi \mathbf{G R}-\mathbf{R}\} \delta \mathbf{R} .
$$

Reference to $(2.15)$ and $\left(2.15^{\prime}\right)$ shows that $\delta \mathfrak{I}$ is indeed zero for the correct $\mathbf{R}$. Conversely if $\mathfrak{I}$ is stationary, the quantities within the brackets must vanish, which yields $(2.15)$ and $\left(2.15^{\prime}\right)$. The stationary value obtained for the correct $\mathbf{R}$ is

$$
\mathfrak{I}=\mathbf{G R}=\mathbf{R} \mathbf{G}=(\mathbf{R}-\mathbf{G}) / i \pi
$$

The above formulation suffers from the defect that simply a change in normalization of the trial function will yield different approximate values of $\mathbf{R}$. A stationary formulation that meets this objection is provided by the expression

$$
\mathfrak{\Im}_{b a}{ }^{\prime}=(\mathbf{G R})_{b a}(\mathbf{R G})_{b a}\left[(\mathbf{R R})_{b a}-i \pi(\mathbf{R G R})_{b a}\right]^{-1}
$$

The verification of the stationary nature of (4.5) is straightforward and will not be given. The stationary value of $\mathfrak{I}^{\prime}$ is again $(\mathbf{R}-\mathbf{G}) / i \pi$.

It is worthwhile to point out the difference between these variational expressions and that given by $\mathrm{Ma}$ and Hsueh. ${ }^{12}$ Their procedure is based on the observation that

$$
M=\sum_{a} \delta\left(E-E_{a}\right)\left[\mathbf{R}^{\dagger}(\mathbf{R}-\mathbf{G}-i \pi \mathbf{G} \mathbf{R})\right]_{a a}
$$

is stationary under arbitrary variations of $\mathbf{R}^{\dagger}$ (but not of $\mathbf{R}$ !). This is a sort of one sided variational principle. The stationary value of $M$ is evidently zero. The method cannot be used for a direct computation of $\mathbf{R}$; one must put in a trial $\mathbf{R}$ with some independent parameters and vary the parameters appearing in $\mathbf{R}^{\dagger}$. We shall comment later on the accuracy of their method in connection with some examples.

It was stated in I that (4.2) and (4.5) were correct only if $\mathbf{G}$ were real and symmetric; our present derivation shows that this assumption is unnecessary. The fact that $\mathbf{R}$ and $\mathbf{G}$ commute had been overlooked. We mention in passing that the quantity

$$
\mathbf{N}=\mathbf{R}^{\dagger} \mathbf{G}+\mathbf{G} \mathbf{R}+i \pi \mathbf{R}^{\dagger} \mathbf{G}^{2}-i \pi \mathbf{G}^{2} \mathbf{R}-\pi^{2} \mathbf{R}^{\dagger} \mathbf{G}^{2} \mathbf{R}-\mathbf{R} \mathbf{R},
$$

is stationary under arbitrary, independent variations of $\mathbf{R}$ and $\mathbf{R}^{\dagger}$ about the correct values. However, the demand of vanishing variation yields the equation for $R$ and $R^{\dagger}$ in iterated form, e.g.,

$$
\mathbf{R}=\mathbf{G}+i \pi \mathbf{G}(\mathbf{R}+i \pi \mathbf{G} \mathbf{R}) .
$$

A normalization independent form of this variational principle is easily derived. The stationary value obtained is $\mathbf{G}^{2}$, consequently this principle must be used much as that of $\mathrm{Ma}$ and Hsueh. We shall make no further use of this result.

A few remarks on the relative accuracy of (4.2) and (4.5) are in order. For example, if one puts for a trial function in (4.2) simply $\mathbf{G}$, the approximate value of $\mathbf{R}$ 
thus obtained is just equivalent to two iterations of (2.15). This has the effect that approximate $S$-matrix computed from this approximate $\mathbf{R}$ is not necessarily unitary. The same procedure as applied to (4.5) does not suffer from this difficulty. The solution thus obtained is not an expansion in $\mathbf{G}$, and in general should give a more accurate answer. We shall in fact see a case where (4.5) gives the exact solution whereas (4.2) gives, as it must, simply two iterations.

A similar development may be carried through for Eq. (2.14) which defines $G$ in terms of $B$. We assert that the energy shell matrix $\mathbf{J}_{b a}$ (i.e., $E_{a}=E_{b}=E$ ) given by

$$
\begin{aligned}
\mathbf{J}_{b a}=P \sum_{\alpha} & \frac{G_{b a}^{\dagger} G_{\alpha a}}{E-E_{\alpha}}+P \sum_{\alpha, \beta} \frac{G_{b \alpha}^{\dagger} B_{\alpha \beta} G_{\beta a}}{\left(E-E_{\alpha}\right)\left(E-E_{\beta}\right)} \\
& -P \sum_{\alpha}\left(G_{b \alpha}^{\dagger} B_{\alpha a}+B_{b \alpha} G_{\alpha a}\right) /\left(E-E_{\alpha}\right)
\end{aligned}
$$

is stationary under arbitrary independent variations of $G$ and $G^{\dagger}$ about the correct values. We have

$$
\begin{aligned}
\delta \mathbf{J}_{b a} & =P \sum_{\alpha} \frac{\delta G_{b \alpha}^{\dagger}}{E-E_{\alpha}}\left\{G_{\alpha a}+P \sum_{\beta} \frac{B_{\alpha \beta} G_{\beta a}}{E-E_{\beta}}-B_{\alpha a}\right\} \\
& +P \sum_{\alpha}\left\{G_{b \alpha}^{\dagger}+P \sum_{\beta} \frac{G_{b \beta} \dagger B_{\beta \alpha}}{E-E_{\beta}}-B_{b \alpha}\right\} \frac{\delta G_{\alpha a}}{E-E_{\alpha}}
\end{aligned}
$$

from which the stationary character of $J$ is evident. The stationary value obtained is

$$
\mathbf{J}_{b a}=-\sum_{\alpha} B_{b \alpha} G_{\alpha a} /\left(E-E_{\alpha}\right)=\mathbf{G}_{b a}-\mathbf{B}_{b a} .
$$

It should be noted that if $E_{a}=E_{b}=E$,

$$
P \sum_{\alpha} G_{b \alpha}^{\dagger} B_{\alpha a} /\left(E-E_{\alpha}\right)=P \sum_{\alpha} B_{b \alpha} G_{\alpha a} /\left(E-E_{\alpha}\right) \text {. }
$$

The normalization independent form is easily found and is given by

$$
\mathbf{J}_{b a}{ }^{\prime}=-\left(G^{\dagger} B\right)_{b a}(B G)_{b a}\left[\left(G^{\dagger} G\right)_{b a}+\left(G^{\dagger} B G\right)_{b a}\right]^{-1},
$$

with matrix multiplication defined by

$$
\left(G^{\dagger} G\right)_{b a}=P \sum_{\alpha} G_{b a}^{\dagger} G_{\alpha a} /\left(E-E_{\alpha}\right) \text {, etc. }
$$

The stationary value of $\mathbf{J}_{b a}{ }^{\prime}$ is again given by (4.9). It should be noted that (4.7) and (4.11) are stationary expressions for $\mathbf{G}$, i.e., on the energy shell, and cannot be used for computation of $G$. This is, of course, no difficulty because it is $\mathbf{G}$ that is needed for insertion into (2.15).

With the simplest trial function, $G=B$, (4.8) yields the equivalent of two iterations of (2.14) or stated otherwise the third Born approximation. The form (4.11), however, does not yield a power series expansion in $B$ and may be expected to give a more reliable result, in general.

In applications, the above variational principles appear to have some advantage over the related ones given by Lippmann and Schwinger ${ }^{6}$ in that instead of having to guess wave functions as trial functions one instead must guess essentially scattering amplitudes. In the case of nucleon-nucleon scattering, say, it seems much simpler to guess a reasonable scattering amplitude than a three dimensional wave function which must be especially accurate in the neighborhood of the potential. (Actually the necessary trial functions are not true scattering amplitudes in that behavior of the energy shell must be assumed.)

Our variational formulations for $G$ and $\mathbf{R}$ have some features in common. They generally are neigher maxima nor minima and, second, they both correspond, in their nonhomogeneous form, to the same degree of approximation in an iteration solution. The homogeneous forms take into account what amounts essentially to the renormalization of the wave function familiar from perturbation theory. The necessary renormalization may be easily understood in the case of scattering by a potential. The iteration procedure adds up the contributions from single scattering scatterings, all with the same initial amplitude, then the subsequent scatterings of these scattered waves. The error made is essentially in the single scattering contributions: Those regions of potential farthest from the incident beam do not feel the full strength of the beam for their first scattering, it having been weakened by earlier scatterings.

\section{APPLICATIONS}

We shall now discuss a number of applications of our variational principles to specific examples. Most of our attention will be confined to (2.15) and the associated variational expressions (4.2) and (4.5), because only in rather pathological cases is it possible to solve (2.14) exactly and thus have reliable comparisons with approximate solutions. There are, on the other hand, a number of exact solutions of (2.15) available, at least for the case where the first nonvanishing approximation for $\mathbf{G}$ is used. In connection with the approximate .solutions to be discussed, it is useful to bear in mind the easily derived result that the condition for the convergence of an iteration solution of (2.15) is

$$
\pi^{2} G_{A m}{ }^{2}<1
$$

where $G_{A m}$ is the largest eigenvalue of $\mathbf{G}$ (see discussion following $\left(2.15^{\prime}\right)$; this result is evident from the eigenfunction expansion given there).

\section{Nonrelativistic Compton Effect ${ }^{13}$}

We limit ourselves to a discussion of (2.15) with the first nonvanishing approximation for G. A more complete investigation is in progress. With this limitation the matrix elements of $\mathbf{G}$ between states of a single photon of momentum $\mathbf{k}$, polarization $\boldsymbol{\varepsilon}_{\lambda}$, electron at rest (neglecting the frequency shift) are given by

$$
\left\langle\mathbf{k} \lambda|\mathbf{G}| \mathbf{k}^{\prime} \lambda^{\prime}\right\rangle=-\left(2 \pi e^{2} / m k\right) \boldsymbol{\varepsilon}_{\lambda} \cdot \varepsilon_{\lambda^{\prime}}
$$

\footnotetext{
${ }^{13}$ See W. Heitler, reference 2.
} 
(here and from now on we use natural units $\hbar=c=1$ ). With our normalization, the density of states per unit energy interval is given by

$$
\rho=k^{2} d \Omega /(2 \pi)^{3},
$$

and the Heitler equation becomes

$$
\begin{aligned}
&\left\langle\mathbf{k} \lambda|\mathbf{R}| \mathbf{k}_{0} \lambda_{0}\right\rangle=-\left(2 \pi e^{2} / m k\right) \boldsymbol{\varepsilon}_{\lambda} \cdot \boldsymbol{\varepsilon}_{\lambda_{0}} \\
&-i \pi \sum_{\lambda^{\prime}} \int d \Omega^{\prime}\left(2 \pi e^{2} / m k\right) \boldsymbol{\varepsilon}_{\lambda} \cdot \boldsymbol{\varepsilon}_{\lambda^{\prime}} \frac{k^{2}}{(2 \pi)^{3}} \\
& \times\left\langle\mathbf{k}^{\prime} \lambda^{\prime}|\mathbf{R}| \mathbf{k}_{0} \lambda_{0}\right\rangle .
\end{aligned}
$$

This equation is readily solved with the "ansatz"

$$
\left\langle\mathbf{k} \lambda|\mathbf{R}| \mathbf{k}^{\prime} \lambda^{\prime}\right\rangle=U(k) \boldsymbol{\varepsilon}_{\lambda} \cdot \boldsymbol{\varepsilon}_{\lambda^{\prime}}
$$

with the result

$$
\begin{aligned}
\left\langle\mathbf{k} \lambda|\mathbf{R}| \mathbf{k}_{0} \lambda_{0}\right\rangle & \\
\quad= & -\left(2 \pi e^{2} / m k\right)\left(1+i\left(2 e^{2} k / 3 m\right)\right)^{-1} \boldsymbol{\varepsilon}_{\lambda} \cdot \boldsymbol{\varepsilon} \lambda_{0} .
\end{aligned}
$$

We now apply (4.5), taking as trial function simply

$$
\left\langle\mathbf{k} \lambda\left|\mathbf{R}_{T}\right| \mathbf{k}_{0} \lambda_{0}\right\rangle=\varepsilon_{\lambda} \cdot \varepsilon_{0} .
$$

The stationary value of $\mathbf{R}$ is, with this trial function precisely the value given by (5.5), the exact answer. The variational method of $\mathrm{Ma}$ and $\mathrm{Hsueh}$ also gives the exact result for this case. The condition for the validity of the iteration solution is evidently $e^{2} k / m<1$ which is certainly true if our neglect of recoil and frequency change are to be justified.

\section{2a. Meson-Nucleon Scattering, $\pi^{+}+N \rightarrow \pi^{+\prime}+N^{\prime}$ (Positive Mesons by Neutrons) ${ }^{14}$}

We again take the first nonvanishing approximation to $G$. With this simplification, the Heitler equation may be solved exactly for all charged meson theories. We take the pseudoscalar theory for purposes of illustration and also to point out an algebraic error in the results of Fukuda and Miyazima, and $\mathrm{Ma}$ and Hsueh. The relevant matrix elements of $\mathbf{G}$ may be written in the barycentric frame as

$$
\left\langle p k|\mathbf{G}| p_{0} k_{0}\right\rangle=\bar{u}\left(a_{1}+a_{2} \gamma_{4}\right) u_{0} / 2 \epsilon_{0}\left(W_{0}^{2}-M^{2}\right),
$$

where $\bar{u}$ and $u_{0}$ are the usual Dirac plane wave spinor amplitudes corresponding to four-momenta $\boldsymbol{p}$ and $\boldsymbol{p}_{0}$ respectively $\left(\bar{u}=u^{*} \gamma_{4}, u^{*} u=1\right), k$ and $k_{0}$ are the final and initial meson four-momenta, respectively, and in our coordinate system, $\mathbf{p}_{0}=-\mathbf{k}_{0}, \mathbf{p}=-\mathbf{k}, \epsilon_{0}=+\left(\mu^{2}\right.$ $\left.+\mathbf{q}^{2}\right)^{\frac{1}{2}}$ with $q$ the magnitude of the momentum of either particle and $\mu$ the meson mass. $W_{0}$ is the total energy of nucleon plus meson.

$$
\begin{array}{ll}
a_{1}=f^{2} M, & g^{2} M\left(M^{2}+3 W_{0}^{2}\right) / \mu^{2} \\
a_{2}=f^{2} W_{0}, & g^{2} W_{0}\left(W_{0}^{2}+3 M^{2}\right) / \mu^{2},
\end{array}
$$

where the terms in $f$ refer to pseudoscalar coupling and

\footnotetext{
${ }^{14}$ S. T. Ma and C. F. Hsueh, Proc. Cambridge Phil. Soc. 40, 167 (1944) and also reference 12.
}

those in $g$ to pseudovector coupling. $a_{2}$ is given incorrectly in both references ${ }^{14}$ but our results agree with those of Ashkin et al. ${ }^{15}$ The Heitler equation is easily solved for this case because aside from the factors $\bar{u}$ and $u_{0}, \mathbf{G}$ does not depend on angles. In terms of

it becomes

$$
\begin{aligned}
\left\langle p k|\mathbf{R}| p_{0} \boldsymbol{k}_{0}\right\rangle & =\left(1 / 2 \epsilon_{0}\right) \tilde{u} \Re u_{0}, \\
\left\langle p \boldsymbol{k}|\mathbf{G}| p_{0} \boldsymbol{k}_{0}\right\rangle & =\left(1 / 2 \epsilon_{0}\right) \bar{u}\left(\mathfrak{S} u_{0},\right.
\end{aligned}
$$

$$
\begin{aligned}
& \mathfrak{R}\left(\boldsymbol{p k} ; \boldsymbol{p}_{0} \boldsymbol{k}_{0}\right)=\mathfrak{S}\left(\boldsymbol{p} \boldsymbol{k} ; \boldsymbol{p}_{0} \boldsymbol{k}_{0}\right) \\
&+\left(i q / 32 \pi^{2} W_{0}\right) \int d \Omega^{\prime}\left(\mathfrak{S}\left(\boldsymbol{p} \boldsymbol{k} ; \boldsymbol{p}^{\prime} \boldsymbol{k}^{\prime}\right)\left(-i \boldsymbol{\gamma} \cdot \boldsymbol{p}^{\prime}+M\right)\right. \\
& \quad \times \mathfrak{R}\left(\boldsymbol{p}^{\prime} \boldsymbol{k}^{\prime} ; \boldsymbol{p}_{0} \boldsymbol{k}_{0}\right) .
\end{aligned}
$$

Since $\boldsymbol{( S}$ does not depend on angles, $\mathfrak{R}$ is proportional to $(\mathfrak{S}$ and the solution is simply

where

$$
\mathfrak{R}=(1-i \lambda)^{-1} \mathfrak{S}
$$

$$
\lambda=\left(q / 8 \pi W_{0}\right)\left(\mathfrak{S}\left(E_{0} \gamma_{4}+M\right)\right.
$$

with $E_{0}$ the nucleon energy and $M$ its mass. This may be rewritten as

$$
\mathfrak{\Re}=\frac{1+i \kappa_{1}+i_{\kappa_{2} \gamma_{4}}}{\left(1+i_{\kappa_{1}}\right)^{2}+\kappa_{2}^{2}} \frac{a_{1}-a_{2} \gamma_{4}}{W_{0}^{2}-M^{2}},
$$

where

$$
\kappa_{1}=\frac{q}{8 \pi W_{0}} \frac{E_{0} a_{2}-M a_{1}}{W_{0}^{2}-M^{2}}, \quad \kappa_{2}=\frac{q}{8 \pi W_{0}} \frac{E_{0} a_{1}-M a_{2}}{W_{0}^{2}-M^{2}} .
$$

The differential cross section may be computed from (5.13) but since we are not interested at this time in the result we shall merely give the total cross section which is most easily obtained from (2.5). This relation becomes, for our problem

$$
\sigma=\left(1 / q W_{0}\right) \frac{1}{4} \operatorname{Im} . \operatorname{trace} \mathfrak{\Re}\left(-i \gamma \cdot p_{0}+M\right)
$$

which is easily evaluated to yield

$$
\sigma=\frac{8 \pi}{q^{2}}\left[\frac{\kappa_{1}^{2}+\kappa_{2}^{2}+\left(\kappa_{2}^{2}-\kappa_{1}^{2}\right)^{2}}{1+2\left(\kappa_{1}^{2}+\kappa_{2}^{2}\right)+\left(\kappa_{2}^{2}-\kappa_{1}^{2}\right)^{2}}\right] .
$$

The criterion for the convergence of an iteration solution may be expressed in terms of the largest eigenvalue of the operator $\lambda$ in (5.12), i.e., $\lambda^{2}<1$. These eigenvalues are easily found and yield the result that for pseudoscalar coupling we must have

$$
\frac{1}{4}\left(\frac{f^{2}}{4 \pi}\right)^{2} \frac{q^{2}}{W_{0}^{2}}\left(\frac{E_{0}+M}{W_{0}+M}\right)^{2}<1
$$

In this case the damping effect is dominated by the size of the coupling constant as may be seen by considering the extreme relativistic limit, in which case the requirement is

$$
\frac{1}{4}\left(f^{2} / 4 \pi\right)^{2}<1
$$

${ }^{15}$ Ashkin, Simon, and Marshak, Prog. Theor. Phys. V, 634 (1950). 
The entire damping effect is much more important in the pseudovector coupling case. We find the convergence requirement

$$
\frac{1}{4}\left(\frac{g^{2}}{4 \pi}\right)^{2} \frac{q^{2}}{W_{0}^{2}}\left(\frac{E_{0}+M}{W_{0}+M}\right)^{2}\left(\frac{W_{0}-M}{\mu}\right)^{4}
$$

from which we see that for very high energies the iteration procedure will certainly fail.

We turn now to the variational treatment. Equation (4.5) may of course be used as it stands, but it is more convenient to write a stationary expression for $\mathfrak{\Re}$. This is

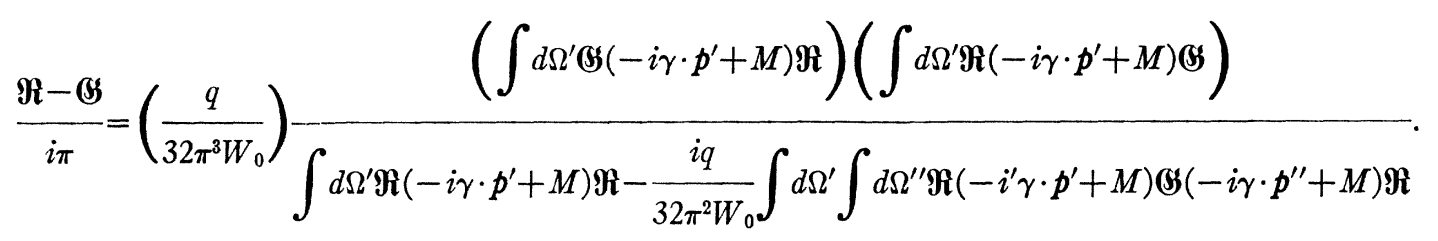

If we take as a trial function $\mathfrak{R}=(\mathfrak{S}$ we find

$$
\begin{aligned}
\mathfrak{R} & \simeq\left(\mathfrak{S}+\frac{\left(i q / q \pi W_{0}\right) \mathfrak{G}\left(E_{0} \gamma_{4}+M\right) \mathfrak{G}}{1-\left(i q / 8 \pi W_{0}\right) \mathfrak{S}\left(E_{0} \gamma_{4}+M\right)}\right. \\
& =\frac{1}{1-i\left(q / 8 \pi W_{0}\right) \mathfrak{G}\left(E_{0} \gamma_{4}+M\right)}
\end{aligned}
$$

which is the exact answer. The same result may be obtained using the inhomogeneous form (4.2) written in terms of $\mathfrak{R}$ and $\mathfrak{S}$ with a trial function $\mathfrak{R}=a+b \gamma_{4}$ where $\left[a, \gamma_{4}\right]=\left[b, \gamma_{4}\right]=0$ in order that $[\mathfrak{R}, \mathfrak{G}]=0$, and $a$ and $b$ independent of angles. It is essential to have adjustable quantities available when using (4.2) in order that the correct normalization be obtained. $\mathrm{Ma}$ and Hsueh have also applied their method to this problem and obtained poor results.

\section{2b. Meson-Nucleon Scattering, $\pi^{-}+N \rightarrow \pi^{-\prime}+N^{\prime}$} (Negative Mesons by Neutrons)

Even with the Heitler approximation for $\mathbb{E}$, the integral Eq. (2.15) cannot be solved exactly in the relativistic case, as has been pointed out in reference 15. (Actually one may obtain the exact solution provided only that the product of $v / c$ for meson and nucleon is small compared with unity.) We shall for simplicity deal only with the non-relativistic case where in addition we neglect the nucleon recoil. We take pseudoscalar mesons with pseudovector coupling. The matrix elements of $\mathbf{G}$ may be regarded as operators on the spin variables of the neutron and are given by

$$
\left\langle\mathbf{k}|\mathbf{G}| \mathbf{k}_{0}\right\rangle=\left(g^{2} / 2 \mu^{2} \epsilon_{0}\right) \boldsymbol{\sigma} \cdot \mathbf{k}_{0} \mathbf{\sigma} \cdot \mathbf{k} .
$$

The Heitler equation becomes

$$
\begin{array}{r}
\left\langle\mathbf{k}|\mathbf{R}| \mathbf{k}_{0}\right\rangle=\left(g^{2} / 2 \mu^{2} \epsilon_{0}\right) \boldsymbol{\sigma} \cdot \mathbf{k}_{0} \boldsymbol{\sigma} \cdot \mathbf{k}+i\left(g^{2} k_{0} / 16 \pi^{2} \mu^{2} \epsilon_{0}\right) \\
\times \int d \Omega^{\prime} \boldsymbol{\sigma} \cdot \mathbf{k}^{\prime} \boldsymbol{\sigma} \cdot \mathbf{k}\left\langle\mathbf{k}^{\prime}|R| \mathbf{k}_{0}\right\rangle .
\end{array}
$$

The solution is easily found by noting that the only scalars that can be constructed from $\mathbf{k}_{0}, \mathbf{k}$ and $\boldsymbol{\sigma}$ are $\mathbf{k}_{0} \cdot \mathbf{k}$ and $\boldsymbol{\sigma} \cdot\left(\mathbf{k}_{0} \times \mathbf{k}\right)$. The solution must consequently be a linear combination of these. One finds

$$
\left\langle\mathbf{k}|\mathbf{R}| \mathbf{k}_{0}\right\rangle=\left(g^{2} / 2 \mu^{2} \epsilon_{0}\right) \frac{(1+2 i x) \mathbf{k}_{0} \cdot \mathbf{k}+i \boldsymbol{\sigma} \cdot \mathbf{k}_{0} \times \mathbf{k}}{1-i x+2 x^{2}}
$$

where

$$
x=\left(g^{2} / 4 \pi\right)\left(k_{0}^{3} / 3 \mu^{2} \epsilon_{0}\right) .
$$

The cross section is easily found to be

$$
\begin{aligned}
& d \sigma=\left(g^{2} / 4 \pi\right)\left(1 / \mu^{2}\right)\left(k_{0}^{4} / \mu^{2} \epsilon_{0}^{2}\right) \\
& \times\left[1+4 x^{2} \cos ^{2} \vartheta\right] /\left[\left(1+2 x^{2}\right)^{2}+x^{2}\right] .
\end{aligned}
$$

If the damping is neglected $(x=0)$ one finds the well known result. It is interesting to note that for very large $x$, the cross section is simply

$$
d \sigma=\left(36 / k_{0}{ }^{2}\right) \cos ^{2} \vartheta d \Omega
$$

independent of $g$ and showing the characteristic $p$ wave angular distribution of scattered mesons.

If one now attempts to solve (5.23) approximately using the trial function $\mathbf{R}=\mathbf{G}$ in (4.5), for example, one obtains a rather complicated result which does however yield the exact cross section plus correction terms of order $x^{8}$. The expression (4.2) with the same trial function would yield only $x^{2}$ terms reliably, indicating the superiority of (4.5) over (4.2). The trial function

$$
\left\langle\mathbf{k} \mid \mathbf{R}_{T} / \mathbf{k}_{0}\right\rangle=\alpha \mathbf{k}_{0} \cdot \mathbf{k}+\beta \boldsymbol{\sigma} \cdot \mathbf{k}_{0} \times \mathbf{k}
$$

with parameters $\alpha$ and $\beta$ determined using the stationary character of (4.2) yields the exact solution, (5.24).

The purpose of these examples has been to show the general reliability of the variational principles. No definite statement as to their accuracy can be made. This is of course frequently the case and is the biggest objection to variational techniques. It might be noted that this uncertainty may work in one's favor as is seen by the last example; statements about the errors in the results of variationally computed quantities in terms of alleged errors in trial functions are generally meaningless. 


\section{Blatt's Example ${ }^{10}$}

It is instructive to study an example given by Blatt in his discussion of the Heitler damping theory. The problem is that of the scattering of a scalar meson by a fixed, extended, source. The hamiltonian is

$$
\begin{aligned}
& H=\frac{1}{2} \int d \mathbf{x}\left[\pi^{2}(\mathbf{x})+\varphi(\mathbf{x})\left(\mu^{2}-\nabla^{2}\right) \varphi(\mathbf{x})\right]+\frac{1}{2} \lambda \bar{\varphi}^{2} \\
& \bar{\varphi}=\int d \mathbf{x} \varphi(\mathbf{x}) U(\mathbf{\sigma}) .
\end{aligned}
$$

This problem has also been treated by Wentzel ${ }^{16}$ in another connection. These authors show that an exact solution may be given in terms of normal coordinates and in particular that the problem of meson scattering reduces to a study of the equation

$$
\left[k_{0}^{2}-\left(-\nabla^{2}\right)\right] \Psi(\mathbf{x})=\lambda \bar{\Psi} U(\mathbf{x}) .
$$

Our general equations may be written in terms of these quantities identifying $E_{a}, H_{0}, H_{1} \Psi$ with $k_{0}^{2},-\nabla^{2}$, $\lambda \bar{\Psi} U(\mathbf{x})$ respectively. The wave number of the scattered meson will be called $\mathbf{k}$, its magnitude being equal to that of the incident meson, $\mathbf{k}_{0}$. The solution for the scattering problem as given by Blatt (slightly rewritten to correspond to quantization in a box of unit volume) is

$$
\begin{aligned}
\Psi_{\mathbf{k}_{0}}(+) & =\Phi \mathbf{k}_{0}-\lambda \bar{\Psi}_{\mathbf{k}_{0}}{ }^{(+)} V\left(k_{0}, \mathbf{x}\right) \\
\Phi_{\mathbf{k}_{0}} & =(2 E)^{-\frac{1}{2}} e^{i \mathbf{k}_{0} \cdot \mathbf{x}} \\
V\left(k_{0}, \mathbf{x}\right) & =\frac{1}{4 \pi} \int d \mathbf{x}^{\prime} \frac{e^{i k_{0}\left|\mathbf{x}-\mathbf{x}^{\prime}\right|}}{\left|\mathbf{x}-\mathbf{x}^{\prime}\right|} U\left(\mathbf{x}^{\prime}\right) \\
\overline{\Psi_{\mathbf{k}_{0}}}(+) & =\int d \mathbf{x} \Psi \mathbf{k}_{0}{ }^{(+)} U(\mathbf{x}) \\
& =v\left(-k_{0}\right)\left[1+\lambda \bar{V}\left(k_{0}\right)\right]^{-1}(2 E)^{-\frac{1}{5}} \\
\bar{V}\left(k_{0}\right) & =\int d \mathbf{x} V\left(k_{0}, \mathbf{x}\right) U(\mathbf{x}) \\
v\left(k_{0}\right) & =\int d \mathbf{x} e^{-i \mathbf{k}_{0} \cdot \mathbf{x}} U(\mathbf{x}) \\
E & =+\left(\mu^{2}+k_{0}{ }^{2}\right)^{\frac{1}{2}} .
\end{aligned}
$$

The scattering amplitude may be obtained from the asymptotic form of $\Psi_{\mathbf{k}_{0}}{ }^{(+)}$which is

$$
\begin{aligned}
\Psi_{\mathbf{k}_{0}}{ }^{(+)}(\mathbf{x}) & \rightarrow(2 E)^{-\frac{3}{3}}\left[e^{i \mathbf{k}_{0} \cdot \mathbf{x}}-\frac{e^{i k_{0}|\mathbf{x}|}}{|\mathbf{x}|} \frac{\lambda}{4 \pi} \frac{\gamma\left(k_{0}\right)}{1+\lambda \bar{V}\left(k_{0}\right)}\right] \\
\gamma\left(k_{0}\right) & =v\left(-k_{0}\right) v\left(k_{0}\right) .
\end{aligned}
$$

We shall now solve this problem in two steps according to our general procedure. It is convenient to

\footnotetext{
${ }^{16}$ G. Wentzel, Helv. Phys. Acta XV, 111 (1942).
}

introduce in place of the matrix $\mathbf{R}$ the usual scattering amplitude $f\left(\mathbf{k}, \mathbf{k}_{0}\right)$; the two are related by

$$
f\left(\mathbf{k}, \mathbf{k}_{0}\right)=\left[E\left(k_{0}\right) / 2 \pi\right]\left\langle\mathbf{k}|\mathbf{R}| \mathbf{k}_{0}\right\rangle .
$$

Similarly we introduce

$$
\begin{aligned}
& g(\mathbf{p}, \mathbf{q})=\left[\{E(p) E(q)\}^{\frac{1}{2}} / 2 \pi\right]\langle\mathbf{p}|G| \mathbf{q}\rangle, \\
& b(\mathbf{p}, \mathbf{q})=\left[\{E(p) E(q)\}^{\frac{1}{2}} / 2 \pi\right]\langle\mathbf{p}|B| \mathbf{q}\rangle,
\end{aligned}
$$

in terms of which (2.14) and (2.15) become, respectively,

$$
\begin{aligned}
& g\left(\mathbf{p}, \mathbf{k}_{0}\right)=b\left(\mathbf{p}, \mathbf{k}_{0}\right)+\frac{P}{2 \pi^{2}} \int d \mathbf{p} \frac{b\left(\mathbf{p}, \mathbf{p}^{\prime}\right) g\left(\mathbf{p}^{\prime}, \mathbf{k}_{0}\right)}{p^{\prime 2}-k_{0}^{2}} \\
& f\left(\mathbf{k}, \mathbf{k}_{0}\right)=g\left(\mathbf{k}, \mathbf{k}_{0}\right)+\frac{i k_{0}}{4 \pi} \int d \Omega^{\prime} g\left(\mathbf{k}, \mathbf{k}^{\prime}\right) f\left(\mathbf{k}^{\prime}, \mathbf{k}_{0}\right)
\end{aligned}
$$

For our particular problem,

$b(\mathbf{p}, \mathbf{q})$

$$
\begin{aligned}
& =-(\lambda / 4 \pi) \int d \mathbf{x} \int d \mathbf{x}^{\prime} e^{-i \mathbf{p} \cdot \mathbf{x}} U(\mathbf{x}) U\left(\mathbf{x}^{\prime}\right) e^{i \mathbf{q} \cdot \mathbf{x}^{\prime}} \\
& =-(\lambda / 4 \pi) v(p) v(-p) .
\end{aligned}
$$

The solution of these equations is trivial by virtue of the separability of $b$. We find for $g$,

$$
\begin{aligned}
& g\left(\mathbf{p}, \mathbf{k}_{0}\right)=g\left(p, k_{0}\right)=-(\lambda / 4 \pi) \frac{v(p) v\left(-k_{0}\right)}{1+\lambda \bar{W}\left(k_{0}\right)} \\
\bar{W}\left(k_{0}\right)= & \frac{P}{(2 \pi)^{3}} \int d \mathbf{p} \frac{\gamma(p)}{p^{2}-k_{0}{ }^{2}} \\
= & \frac{1}{4 \pi} \int d \mathbf{x} \int d \mathbf{x}^{\prime} U(\mathbf{x}) \frac{\cos k_{0}\left|\mathbf{x}-\mathbf{x}^{\prime}\right|}{\left|\mathbf{x}-\mathbf{x}^{\prime}\right|} U\left(\mathbf{x}^{\prime}\right) .
\end{aligned}
$$

With this value for $g$ we have immediately

$$
f\left(\mathbf{k}, \mathbf{k}_{0}\right)=-\frac{\lambda}{4 \pi} \frac{\gamma\left(k_{0}\right)}{1+\lambda\left(\bar{W}\left(k_{0}\right)+i k_{0} \gamma\left(k_{0}\right) / 4 \pi\right)} .
$$

That this is the same as Blatt's solution follows from the fact that

$$
\bar{V}\left(k_{0}\right)=\frac{1}{(2 \pi)^{3}} \int d \mathbf{p} \frac{\gamma(p)}{p^{2}-k_{0}{ }^{2}-i \epsilon}=\bar{W}\left(k_{0}\right)+\frac{i k_{0} \gamma\left(k_{\theta}\right)}{4 \pi} .
$$

The usual damping theory prescription of taking the first nonvanishing term in the iteration solution corresponds to putting $\bar{W}$ equal to zero. Blatt's failure to recognize the source function $\gamma\left(k_{0}\right)$ in the damping term, $i k_{0} \gamma\left(k_{0}\right) / 4 \pi$, of the exact solution was a result of his configuration space expansion of $\bar{V}\left(k_{0}\right)$; the only error in the usual Heitler method is the setting of $\bar{W}$ equal to zero. As pointed out by Blatt, the seriousness 
of this error depends essentially on numerical values. For small values of $k_{0}, \bar{W}\left(k_{0}\right)$ is related to the source radius, $a$, according to

$$
\bar{W}\left(k_{0}\right) \simeq \frac{1}{4} \pi a,
$$

so that the ratio of the damping to the inertial term in (5.38) is approximately $k_{0} a$ which is by hypothesis very small. Evidently then the neglect of $\bar{W}\left(k_{0}\right)$ is unjustified. On the other hand this dominant term in $\bar{W}\left(k_{0}\right)$ is definitely a nucleon self-energy term which should be incorporated into the nucleon mass in the spirit of modern renormalization. For this simple problem it may also be regarded as a renormalization of the coupling parameter $\lambda$. To show this explicitly we write

$$
\begin{aligned}
\bar{W}\left(k_{0}\right)=\frac{1}{4 \pi a}+\frac{1}{4 \pi} \int d \mathbf{x} \int & d \mathbf{x}^{\prime} U(\mathbf{x}) \\
& \times \frac{\cos k_{0}\left|\mathbf{x}-\mathbf{x}^{\prime}\right|-1}{\left|\mathbf{x}-\mathbf{x}^{\prime}\right|} U\left(\mathbf{x}^{\prime}\right) .
\end{aligned}
$$

Calling the second term in (5.40) $\bar{w}\left(k_{0}\right)$ we may rewrite (5.36) as

$$
\begin{aligned}
g\left(p, k_{0}\right)=-\frac{\lambda}{4 \pi}(1+\lambda / 4 \pi a)^{-1} & \\
& \times \frac{v(p) v\left(-k_{0}\right)}{1+\lambda(1+\lambda / 4 \pi a)^{-1} \bar{w}\left(k_{0}\right)} .
\end{aligned}
$$

Making the obvious replacement $\lambda(1+\lambda / 4 \pi a)^{-1} \rightarrow \lambda^{\prime}$, we have

$$
\begin{aligned}
& g\left(p, k_{0}\right)=-\left(\lambda^{\prime} / 4 \pi\right) v(p) v\left(-k_{0}\right) /\left(1+\lambda^{\prime} \bar{w}\left(k_{0}\right)\right) \\
& f\left(\mathbf{k}, \mathbf{k}_{0}\right)=-\left(\lambda^{\prime} / 4 \pi\right) \frac{\gamma\left(k_{0}\right)}{1+\lambda^{\prime}\left[\bar{w}\left(k_{0}\right)+i k_{0} \gamma\left(k_{0}\right) / 4 \pi\right]}
\end{aligned}
$$

The question of the legitimacy of neglecting $\bar{w}\left(k_{0}\right)$ depends still on numerical values. If for example one now passes to the limit of a point interaction $\left[v\left(k_{0}\right) \rightarrow 1\right] \bar{w}\left(k_{0}\right)$ is identically zero, and the Heitler prescription would be exact. If the source is very small, but still finite, an iteration solution for $g$ would be feasible in an energy range for which iteration for $f$ would fail. For example if we take a gaussian shape for $U(\mathbf{x})$ and $k_{0} a \ll 1$, we find

$$
\left|4 \pi \bar{w}\left(k_{0}\right) / k_{0} \gamma\left(k_{0}\right)\right| \simeq 2 k_{0} a / \pi \ll 1,
$$

and the iteration solution for $f$ fails if $k_{0} \lambda \gg 1$. For $k_{0} a \gg 1$, on the other hand,

$$
\left|4 \pi \bar{w}\left(k_{0}\right) / k_{0} \gamma\right| \simeq e^{k_{0}^{2} a^{2} / \pi} / k_{0} a \gg 1,
$$

in which case the iteration for $g$ would not be permissible (unless $\lambda / a \gg 1$ ) whereas that for $f$ would be convergent. The general situation in a theory of point particles is probably that damping terms are dominant, after renormalization of the inductive terms, but this question has not been thoroughly investigated as yet.
Almost identical discussions may be given for the other examples treated by Blatt so there is little point in presenting them.

\section{ADDITIONAL FORMAL CONSIDERATIONS}

It was mentioned briefly in Sec. II that one may formally solve the Heitler Eq. (2.15) in terms of the eigenfunctions of the operator $\mathbf{G}$. It is useful also to obtain a formal solution to (2.14) which we shall refer to as the reaction equation. We begin by studying the eigenvalue problem defined by

$$
\sum_{\alpha} B_{\beta \alpha} u_{\Gamma}(\alpha) /\left(E_{a}-E_{\alpha}\right)=B_{\Gamma}\left(E_{a}\right) u_{\Gamma}(\beta)
$$

with the normalization condition.

$$
\sum_{\alpha} u_{\Gamma}{ }^{*}(\alpha) u_{\Gamma^{\prime}}(\alpha) /\left[E_{a}-E_{\alpha}\right]=\epsilon \delta_{\Gamma \Gamma^{\prime}} .
$$

(Principle values are implied in the above integrals and in the subsequent ones.) The desired eigenfunction $u_{\Gamma}$ must be normalized in the unconventional manner shown in (6.2) in order that (6.1) be an hermitian eigenvalue problem. The quantity $E_{a}$ is a base energy which is conveniently chosen as the same energy appearing in (2.14) which we rewrite for ease of reference:

$$
G_{\beta a}=B_{\beta a}-\sum_{\alpha} B_{\beta \alpha} G_{\alpha a} /\left(E_{a}-E_{\alpha}\right) \text {. }
$$

The quantity $\epsilon$, which is the value of the normalization integral may be chosen as \pm 1 if desired; the particular sign is not a priori known. It follows from (6.1) and (6.2) that

$$
B_{\beta \alpha}=\epsilon^{-1} \sum_{\Gamma} u_{\Gamma}(\beta) B_{\Gamma} u_{\Gamma}^{*}(\alpha),
$$

as may be verified by noting that multiplication of (6.4) by $u_{\Gamma^{\prime}}(\alpha) / E_{a}-E_{\alpha}$ and summation over $\alpha$, together with (6.2) yields (6.1). The analogous operation performed on (6.1) shows the reality of the $B_{\Gamma}\left(E_{a}\right)$.

In terms of the eigenfunctions $u_{\Gamma}(\alpha)$ the solution of the reaction equation is very simply obtained. Substituting (6.4) into (6.3) we have

$$
\begin{aligned}
G_{\beta a}=\frac{1}{\epsilon} \sum_{\Gamma^{\prime}} u_{\Gamma^{\prime}}(\beta) B_{\Gamma^{\prime}} u_{\Gamma^{\prime}} *(a) & \\
& -\frac{1}{\epsilon} \sum_{\alpha} \sum_{\Gamma^{\prime}} \frac{u_{\Gamma^{\prime}}(\beta) B_{\Gamma^{\prime}} u_{\Gamma^{\prime}} *(\alpha) G_{\alpha a}}{E_{a}-E_{\alpha}} .
\end{aligned}
$$

Multiplication by $u_{\Gamma}{ }^{*}(\beta) /\left(E_{a}-E_{\beta}\right)$ and summation over $\beta$ yields, using (6.2),

$$
\begin{gathered}
\sum_{\beta} \frac{u_{\Gamma}^{*}(\beta) G_{\beta a}}{E_{a}-E_{\beta}}=B_{\Gamma} u_{\Gamma}^{*}(a)-B_{\Gamma} \sum_{\beta} \frac{u_{\Gamma}^{*}(\beta) G_{\beta a}}{E_{a}-E_{\beta}} \\
\sum_{\beta} \frac{u_{\Gamma}^{*}(\beta) G_{\beta a}}{E_{a}-E_{\beta}}=\frac{B_{\Gamma} u_{\Gamma}^{*}(a)}{1+B_{\Gamma}}
\end{gathered}
$$

or

Substituting (6.7) into (6.5) yields immediately

with

$$
G_{\beta a}=\epsilon^{-1} \sum_{\Gamma} u_{\Gamma}(\beta) G_{\Gamma}\left(E_{a}\right) u_{\Gamma}^{*}(a),
$$

$$
G_{\Gamma}\left(E_{a}\right)=B_{\Gamma}\left(E_{a}\right) /\left[1+B_{\Gamma}\left(E_{a}\right)\right]
$$


The dependence of $G_{\mathrm{r}}$ on $E_{a}$ shows again the fact that $G$ is not an hermitian matrix [see (2.12)]. An iteration solution of the reaction equation evidently corresponds to an expansion of the denominator of (6.9) from which we obtain the convergence requirement

$$
\left|B_{\Gamma}\left(E_{a}\right)\right|<1 \text {. }
$$

It is worth while noting that the expression

$$
B_{\Gamma}=\left[\sum_{\alpha, \beta} \frac{u_{\Gamma}^{*}(\beta) B_{\beta \alpha} u_{\Gamma}(\alpha)}{\left(E_{a}-E_{\beta}\right)\left(E_{a}-E_{\alpha}\right)}\right]\left[\sum_{\alpha} \frac{u_{\Gamma}^{*}(\alpha) u_{\Gamma}(\alpha)}{E_{a}-E_{\alpha}}\right]^{-1}
$$

is stationary under arbitrary variations of $u_{\Gamma}$ and $u_{\Gamma}{ }^{+}$.

There is a close connection between the eigenvalue $G_{\Gamma}$ and the $G_{A}$ mentioned in Sec. II in that on the energy shell the eigenfunctions $u_{\Gamma}(a)$ (or more properly, their energy shell projection) and the $f_{a A}$ both provide a diagonalization of $\mathbf{G}$. The formal connection may be written as follows. In terms of the $f_{a A}$, the eigenvalue problem of Sec. II is

$$
\begin{aligned}
\sum_{c} \mathbf{G}_{b c} \delta\left(E-E_{c}\right) f_{c A} & =G_{A} f_{b A}, \\
\sum_{c} f_{c A^{*}} \delta\left(E-E_{c}\right) f_{c A^{\prime}} & =\delta_{A A^{\prime}} .
\end{aligned}
$$

In terms of the transformation coefficient

$$
\langle\Gamma / A\rangle=\sum_{c} u_{\Gamma}^{*}(c) \delta\left(E-E_{c}\right) f_{c A},
$$

we find, introducing (6.8) for $G_{b c}$, that

$$
f_{b A}=\left(\epsilon G_{A}\right)^{-1} \sum_{\mathrm{\Gamma}} u_{\Gamma}(b) G_{\Gamma}\langle\Gamma / A\rangle,
$$

where $\langle\Gamma / A\rangle$ is found from the solution of the set of equations

$$
\begin{aligned}
& \epsilon^{-1} \sum_{\Gamma^{\prime}}\left\langle\Gamma / \Gamma^{\prime}\right\rangle G_{\Gamma^{\prime}}\left\langle\Gamma^{\prime} / A\right\rangle=G_{A}\langle\Gamma / A\rangle \\
& \left\langle\Gamma / \Gamma^{\prime}\right\rangle=\sum_{c} u_{\Gamma}{ }^{*}(c) \delta\left(E-E_{c}\right) u_{\Gamma^{\prime}}(c) .
\end{aligned}
$$

The solution of (6.15) then yields also the eigenvalues $G_{A}$ in terms of the $G_{\Gamma}$. The normalization condition of (6.12) may be expressed in terms of the $\langle\Gamma / A\rangle$ as

$$
\epsilon^{-1} \sum_{\Gamma}\left\langle A^{\prime} / \Gamma\right\rangle G_{\Gamma}\langle\Gamma / A\rangle=G_{A^{\prime}} \delta_{A^{\prime} A} .
$$

A very simple illustration of the formal procedure is furnished by a treatment of Blatt's example. In terms of the notation used in the earlier discussion of the problem, (6.1) becomes

$$
\begin{aligned}
-\left(1 / 2 \pi^{2}\right) \int d \mathbf{q}\left[b(\mathbf{p}, \mathbf{q}) u_{\Gamma}(\mathbf{q})\right] /[ & \left.q^{2}-k_{0}{ }^{2}\right] \\
& =B_{\Gamma}\left(k_{0}{ }^{2}\right) u_{\Gamma}(\mathbf{p}) .
\end{aligned}
$$

Inserting the explicit value of $b(\mathbf{p}, \mathbf{q}),(5.35)$,

$$
\frac{\lambda v(p)}{(2 \pi)^{3}} \int d \mathbf{q} \frac{v(-q) u_{\Gamma}(\mathbf{q})}{q^{2}-k_{0}^{2}}=B_{\Gamma}\left(k_{0}^{2}\right) u_{\Gamma}(\mathbf{p})
$$

The structure of this equation shows that there is only one eigenvalue and one eigenfunction which is simply proportional to $v(p)$. The proportionality factor is obtained from the normalization condition (6.2) which becomes simply

$$
\frac{4 \pi}{(2 \pi)^{3}} \int d \mathbf{q} \frac{u^{*}(\mathbf{q}) u(\mathbf{q})}{q^{2}-k_{0}^{2}}=\epsilon .
$$

In terms of the quantity $\bar{W}\left(k_{0}\right)$ defined in (5.36) we have

$$
\begin{aligned}
B\left(k_{0}^{2}\right) & =\lambda \bar{W}\left(k_{0}\right) \\
u(\mathbf{q}) & =\left(\epsilon /-4 \pi \bar{W}\left(k_{0}\right)\right)^{\frac{1}{2}} v(q)
\end{aligned}
$$

and from (6.8) and (6.9) we obtain our previous answer for $G\left(p, k_{0}\right)$, namely, (5.36). The energy shell eigenfunction of $\mathbf{G}\left(k, k_{0}\right)$ is simply $\left(4 \pi / k_{0}\right)^{\frac{1}{2}}$. The transformation coefficient $\langle\Gamma / A\rangle$ is $\left(4 \pi k_{0}\right)^{\frac{1}{2}} u^{*}\left(k_{0}\right)$, and $G_{A}$ is given in terms of $G_{\Gamma}$ as follows

$$
\begin{aligned}
G_{\Gamma}\left(k_{0}{ }^{2}\right) & =\lambda \bar{W}\left(k_{0}\right) /\left[1+\lambda \bar{W}\left(k_{0}\right)\right] \\
G_{A} & =\left(k_{0} / \epsilon\right) u\left(k_{0}\right) G_{\Gamma} u^{*}\left(k_{0}\right) \\
& =-(\lambda / 4 \pi) k_{0} \gamma\left(k_{0}\right) /\left[1+\lambda \bar{W}\left(k_{0}\right)\right] .
\end{aligned}
$$

Eigenfunction expansions of the above variety, while useful theoretically, are useful in practice only if a few eigenfunctions are needed. In many cases it is preferable to have an approximate closed expression in place of an exact solution in series form. A familiar example is high energy nucleon nucleon scattering and the clumsiness of a phase shift expansion. It is hoped that the variational methods discussed earlier may provide a practical solution in some cases.

Note added in proof.-An alternative variational formulation of the problem of finding $\mathbf{R}$ may be given as follows. Introduce

$$
\begin{aligned}
& R_{\beta \alpha}{ }^{(+)}=-\left(\Phi_{\beta}, H_{1} \Psi_{\alpha}^{(+)}\right) \\
& R_{\beta \alpha}{ }^{(-)}=-\left(\Psi_{\beta}^{(-)}, H_{1} \Phi_{\alpha}\right)
\end{aligned}
$$

which are equal if $E_{\beta}=E_{\alpha}$. These quantities satisfy the equations

$$
\begin{aligned}
& R_{\beta \alpha}{ }^{(+)}=B_{\beta \alpha}-\sum_{\gamma} B_{\beta \gamma} \frac{1}{E_{\alpha}-E_{\gamma}+i \epsilon} R_{\gamma \alpha}^{(+)} \\
& R_{\beta \alpha}{ }^{(-)}=B_{\beta \alpha}-\sum_{\gamma} R_{\beta \gamma}{ }^{(-)} \frac{1}{E_{\beta}-E_{\gamma}+i \epsilon} B_{\gamma \alpha} .
\end{aligned}
$$

It can be verified by direct computation that the energy shell matrix $\mathbf{F}_{b a}$ (i.e., $E_{a}=E_{b}=E$ ) defined by

$$
\begin{aligned}
\mathbf{F}_{b a}= & \sum_{\alpha} \frac{B_{b \alpha} R_{\alpha a}{ }^{(+)}}{E-E_{\alpha}+i \epsilon}+\sum_{\alpha} \frac{R_{b \alpha}(-) B_{\alpha a}}{E-E_{\alpha}+i \epsilon} \\
& -\sum_{\alpha, \beta} \frac{R_{b \beta}(-) B_{\beta \alpha} R_{\alpha a}{ }^{(+)}}{\left(E-E_{\beta}+i \epsilon\right)\left(E-E_{\alpha}+i \epsilon\right)}-\sum_{\alpha} \frac{R_{b \alpha}{ }^{(-)} R_{\alpha a}{ }^{(+)}}{E-E_{\alpha}+i \epsilon}
\end{aligned}
$$

is stationary under arbitrary independent variations of $R^{(+)}$and $R^{(-)}$about the correct values given by (A-2). The stationary value is

$$
R_{b a}^{(+)}-B_{b a}=R_{b a}^{(-)}-B_{b a} .
$$

A normalization independent formulation is easily given. Furthermore, the operator $F$ of which (A-3) is the energy shell matrix element, $\left(\Phi_{b} F \Phi_{a}\right)$ may be explicitly constructed. This result will be discussed in a subsequent publication. 